\title{
INFLUENCIA DE LAS REDES SOCIALES VIRTUALES EN LA CULTURA DE LOS ALUMNOS DE LA UNIVERSIDAD NACIONAL AGRARIA LA MOLINA
}

\author{
SOCIAL NETWORKS INFLUENCE ON THE STUDENT'S CULTURE OF THE \\ UNIVERSIDAD NACIONAL AGRARIA LA MOLINA
}

\author{
${ }^{1}$ Tomás Carlos M. Barriga Barriga
}

\begin{abstract}
Resumen
La presente investigación pretende determinar la influencia del uso de las redes sociales virtuales sobre los valores culturales de los estudiantes de la Universidad Nacional Agraria la Molina. Siendo innumerables los valores culturales potenciales para el estudio, hemos considerado, solamente, la influencia en el lenguaje oral, el lenguaje escrito, el nivel de información, el contacto social, la percepción de popularidad y los valores éticos.
\end{abstract}

Palabras Claves: redes sociales, valores culturales, lenguaje oral, lenguaje escrito, nivel de información, contacto social, popularidad percibida, valores éticos.

\begin{abstract}
The current investigation's purpose is to determinate the influence of the use of social networks on the Universidad Nacional Agraria la Molina students' cultural values. With the fact being that the potential cultural values are uncountable, we have considered, only, the influence on the oral and written language, levels of information, social interaction, perception of popularity and ethical values.
\end{abstract}

Key words: social networks, cultural values, oral language, written language, level information, social contact, perceptions of popularity, ethical values.

\section{Introducción}

Las redes sociales virtuales son sistemas que permiten establecer relaciones entre usuarios a los que se puede conocer o no en la realidad. Cada una de estas relaciones da acceso, además, a todas las personas que tienen algún vínculo con cada usuario, lo cual permite a estas relaciones crecer ilimitadamente en una telaraña de conexiones.

Las Redes son sistemas abiertos, formas de interacción social, definida como un intercambio dinámico entre personas, grupos e instituciones en contextos de complejidad. Un sistema abierto y en construcción permanente que involucra a conjuntos que se identifican en las mismas necesidades y problemáticas y que se organizan para potenciar sus recursos.

En estas comunidades, un número inicial de participantes envían mensajes a miembros de su propia red social invitándoles a unirse al sitio. Los nuevos participantes repiten el proceso, creciendo el número total de miembros y los enlaces de la red. Los sitios ofrecen características como actualización automática de direcciones, perfiles visibles, la capacidad de crear nuevos enlaces mediante servicios de presentación y otras maneras de conexión social en línea. Las redes sociales propician la abolición de los contactos interpersonales, que terminan sustituidos por relaciones ilusorias.

La presente investigación establece la influencia del uso de las redes sociales sobre los valores culturales de los jóvenes estudiantes de la Universidad Nacional Agraria La Molina, de la misma forma, analiza las relaciones entre las redes sociales y la cultura de los jóvenes, teniendo en cuenta que "...entre las nuevas generaciones de nativos digitales se impone un modelo de consumo basado en la información fragmentada", para proponer mejoras en los métodos de acceso a los mismos, en un nivel académico e interpersonal.

El problema principal de la investigación queda planteado con la pregunta ¿De qué manera el uso de las redes sociales virtuales influye en los valores culturales de los estudiantes de la Universidad Nacional Agraria la Molina?

Los problemas específicos tratarán de establecer ¿De qué manera la frecuencia del uso de las redes sociales virtuales, la duración de las sesiones, y el uso principal de una determinada aplicación, influyen en los valores culturales de los estudiantes de la Universidad Nacional Agraria la Molina?

Frente a la realidad expuesta, la presente investigación se realizó dentro del Campus del La Universidad Nacional Agraria la Molina en los años 2012 y 2013, por lo tanto queda circunscrita a la Universidad Nacional Agraria la Molina y a su comunidad estudiantil.

Las redes sociales se han convertido en una herramienta de los jóvenes estudiantes, que principalmente las usan con fines de información y entretenimiento, "una revolución sin precedentes cuyas consecuencias aún no 
podemos prever", por ello es valioso medir su grado de influencia con el fin de poder planificar estrategias que incorporen estos recursos a la actividad docente.

\section{Materiales y métodos}

Para el trabajo se planteó como hipótesis general que el uso de las redes sociales virtuales influye significativamente en los valores culturales de los estudiantes de la Universidad Nacional Agraria La Molina.

Se tomó como Variable Independiente (X) El uso de las redes sociales virtuales; y como Variable dependiente (Y) a los valores culturales.

Se estableció que los indicadores de la variable independiente serían X1: Frecuencia del uso, X2:
Duración de las sesiones, y X3: Uso principal de una aplicación. Por otro lado, se determinó que la variable dependiente tuviera como indicadores a Y1: Lenguaje oral, Y2: Lenguaje escrito, Y3: Nivel de información, Y4: Contacto social, Y5: Popularidad percibida y Y6: Valores éticos.

La presente investigación es de tipo BÁSICA ó PURA, ya que tiene como finalidad el mejor conocimiento de los fenómenos en cuestión. Es a la vez DESCRIPTIVA, ya que trata de informar el estado actual de los fenómenos, cuyos objetivos de estas investigaciones son: indicar los rasgos más saltantes y diferenciarlos y éstas investigan hechos o sucesos ya producidos. La principal característica de estos estudios, consiste en efectuar descripciones minuciosas del tema en estudio.

Tabla 1. Dimensiones, indicadores e índices de la variable independiente: uso de las redes sociales virtuales de los alumnos de la UNALM.

\begin{tabular}{|c|c|c|}
\hline Dimensiones & Indicadores & Items e indices \\
\hline $\begin{array}{l}\text { I. Frecuencia del } \\
\text { uso }\end{array}$ & Items $\mathrm{N}^{\mathrm{o}} \mathrm{s}: 1,2,11$. & Items: $\mathrm{I}=5$ \\
\hline $\begin{array}{l}\text { II. Duración de } \\
\text { las sesiones. }\end{array}$ & Items $\mathrm{N}^{\mathrm{o}} \mathrm{s} 3$. & $\begin{array}{l}\text { 1.¿Cuál es la red social que más usas? } \\
2 . ¿ \text { ¿Cuantos días a la semana ingresas a tu red social favorita aproximadamente? }\end{array}$ \\
\hline $\begin{array}{l}\text { III. Uso principal } \\
\text { de una aplicación. }\end{array}$ & Items $\mathrm{N}^{\mathrm{o}} \mathrm{s}: 4$. & $\begin{array}{l}\text { 3.El día que ingresas a tu red social favorita ¿Cuántas veces lo haces } \\
\text { aproximadamente? } \\
\text { 4.¿Qué aplicación, de tu red social favorita, es la que usas más? } \\
\text { 11. Crees que el tiempo que utilizas en tu red social favorita es: }\end{array}$ \\
\hline
\end{tabular}

Tabla 2. Dimensiones, indicadores e índices de la variable dependiente: influencia de las redes sociales en la cultura de los jóvenes de la UNALM.

\begin{tabular}{ccc}
\hline Dimensiones & Indicadores & Items e indices \\
\hline I. Influencia en lenguaje oral & items $\mathrm{N}^{\circ}$ s: $5,12$. & Items: $1=12$. \\
Indices $=$
\end{tabular}
II. Influencia en el lenguaje Items $N^{\circ} \mathrm{S} 6,13 . .5 . ¿$ La red social que usas ha influido en tu lenguaje oral?
escrito

III. Influencia en el nivel de Items $\mathrm{N}^{\circ} \mathrm{s}: 7,14$. 6.¿La red social que usas ha influido en tu lenguaje escrito?
información.

IV. Influencia en el contacto social Items $\mathrm{N}^{\mathrm{o}} \mathrm{s}: 8,15$. 7.¿Piensas que las redes sociales que usas aumentan tu nivel de información? V. Inlfuencia en la popularidad Items $\mathrm{N}^{\circ} \mathrm{s}: 9,17$. 8.¿Piensas que tus relacione sociales han crecido gracias a tu red social?
percibida

9.¿Cuántos amigos o seguidores tienes afiliados a tu cuenta en tu red social? 10. ¿la información, datos o fotografías de tu red social han influido en tus valores éticos?

12.¿Has aprendido palabras nuevas en tu red social?

13. ¿Cuándo escribes fuera de las redes sociales, utilizas las abreviaturas, signos o emoticones que has aprendido en ellas?

Influencia en los valores éticos. Items $\mathrm{N}^{\mathrm{o}} \mathrm{s}: 10,16.14$. ¿ Te enteras de novedades y datos interesantes a través de tu red social favorita?

15. ¿sientes que gracias a tu red social favorita puedes relacionarte con más personas?

16.¿Has aprendido valores en tu red social, como solidaridad, amistad o algún otro sentimiento noble y deseable?

17.¿A quienes aceptas en tu red social? 
La investigación responde a un diseño de estudio descriptivo, observacional con sistemas de medición, perteneciente a la clase de diseño de un estudio sin intervención. También se le conoce como un diseño No experimental de tipo Transversal o Transaccional ya que tiene como propósito describir las variables y analizar la incidencia e interrelación en un momento dado.

La población está constituida por 4735 alumnos, de las 08 facultades de la Universidad Nacional Agraria La Molina. La Muestra estadísticamente representativa, de la presente investigación, está dada por la aplicación de la fórmula estadística, que corresponde a Arkín y Kolton, considerando un margen de error de $\pm 5 \%$, resulta un Tamaño de Muestra de 339 alumnos.

Tabla 3.Población y muestra del estudio facultades que tiene la Universidad Nacional Agraria La Molina.

\begin{tabular}{lcc}
\hline \multicolumn{1}{c}{ Carrera profesional } & Matriculados & Muestra \\
\hline 1.1 Agronomía & 765 & 55 \\
1.2 Ciencias & 655 & 48 \\
1.3 Ciencias Forestales & 418 & 36 \\
1.4 Economía y Planificación & 1084 & 84 \\
1.5 Industrias Alimentarias & 542 & 21 \\
1.6 Ingeniería agrícola & 401 & 42 \\
1.7 Pesquería & 354 & 24 \\
1.8 Zootecnia & 516 & 32 \\
\hline \multicolumn{1}{c}{ Totales } & 4735 & 342 \\
\hline
\end{tabular}

Para obtener los datos para presente investigación se utilizó la técnica de la Encuesta, con su instrumento el test, que fue aplicado a los estudiantes. Este test se ha tabulado para obtener los datos requeridos.

Otra técnica utilizada es la de Opinión de Expertos, para validar el Cuestionario que aplicamos a los alumnos universitarios para diagnosticar la influencia de las redes sociales en sus valores culturales.

Por último, sabemos que los instrumentos son los medios auxiliares o mecanismos para recoger y registrar los datos obtenidos a través de los métodos y técnicas. En la presente Investigación se empleóun cuestionario tipo test para diagnosticar el nivel de influencia de las redes sociales en los valores culturales de los estudiantes de la Universidad Nacional Agraria la Molina.

Los resultados de las evoluciones, recogidas en las fichas de recolección de datos, fueron pasados a un sistema informático tipo base de datos, se presentarán los resultados en tablas.

El proceso a seguir en el tratamiento de datos fue el siguiente:

a) Distribución porcentual de los datos en cuadros estadísticos:

Se realiza una distribución de los datos en cuadros de distribución de frecuencias de doble entrada, los que sirven para determinar los porcentajes en cada una de las categorías establecidas en los Instrumentos de medición. b) Interpolación de gráficos:

Se realiza una interpolación de los datos en gráficos de barras, los cuales son de mayor comprensión y sencillez para el entendimiento de la naturaleza de los resultados.

Tabla 4. Test para diagnosticar el nivel de influencia de las redes sociales en los valores culturales de los estudiantes de la Universidad Nacional Agraria la Molina

INSTRUCCIONES: Estimado alumno, el presente instrumento, tiene el propósito de recopilar información sobre tus hábitos de uso de las redes sociales virtuales. En la primera parte indagamos algunos aspectos que nos permitan caracterizar las unidades muestrales y en la segunda presentamos una serie de ítems que corresponden propiamente al test.

Mucho te agradeceremos seleccionar la opción y marcar con una " $X$ " en el paréntesis o en el recuadro respectivo y/o complete la información solicitada; el procesamiento será reservado, por lo que te pedimos SINCERIDAD en las respuestas.

I. Caracterización de las unidades muestrales.

SEXO: O Masculino O Femenino

EDAD:

II. Test de medición de la influencia de las redes sociales

1. ¿Cuál es la red social que más usas?

O Facebook O Twitter O Hi5 Olinkedin O NetlogO otra

2. ¿Cuantos días a la semana ingresas a tu red social favorita aproximadamente?

O 1 a 2 días O 3 a 4 días O 5 a 6 días

O todos los días

3. El día que ingresas a tu red social favorita

¿Cuántas tiempo lo haces aproximadamente?

$\mathrm{O}$ menos de 1 hora $\mathrm{O} 1$ a 2 horas $\mathrm{O} 3$ a 4 horas $\mathrm{O} 5$ a

6 horas $\mathrm{O}$ más de 6 horas

3.¿Qué aplicación, de tu red social favorita, es la que usas más?

$\mathrm{O}$ ver fotos $\mathrm{O}$ responder mensajes $\mathrm{O}$ ver videos $\mathrm{O}$ eventos $\mathrm{O}$ curiosear

$\mathrm{O}$ otros

4.¿La red social que usas ha influido en tu lenguaje oral?

$\mathrm{O}$ mucho $\mathrm{O}$ regular $\mathrm{O}$ poco $\mathrm{O}$ nada en absoluto 5.¿La red social que usas ha influido en tu lenguaje escrito?

$\mathrm{O}$ mucho $\mathrm{O}$ regular $\mathrm{O}$ poco $\mathrm{O}$ nada en absoluto 6.¿Piensas que las redes sociales que usas aumentan tu nivel de información?

$\mathrm{O}$ mucho $\mathrm{O}$ regular $\mathrm{O}$ poco $\mathrm{O}$ nada en absoluto 7.¿Piensas que tus relacione sociales han crecido gracias a tu red social?

$\mathrm{O}$ mucho $\mathrm{O}$ regular $\mathrm{O}$ poco $\mathrm{O}$ nada en absoluto

8.¿Cuántos amigos o seguidores tienes afiliados a tu cuenta en tu red social?

$\mathrm{O}$ menos de $50 \mathrm{O}$ entre 50 y $200 \mathrm{O}$ entre 200 y 600

$\mathrm{O}$ entre 600 y 1000

\section{Resultados y discusión}

Se realizó el test instrumental en la Universidad Nacional Agria la Molina durante los semestres 2012-2 y 20131 , a continuación se mostrarán los resultados estadísticos 
porcentuales y los comentarios pertinentes en cada caso: Como se aprecia en la Fig. 1, todos los alumnos encuestados tienen acceso permanente a las redes sociales, encontrando que el $97 \%$ de los jóvenes prefieren el uso de Facebook. Es interesante notar que esta red social está orientada precisamente a la socialización de jóvenes, el intercambio de fotografías e información.

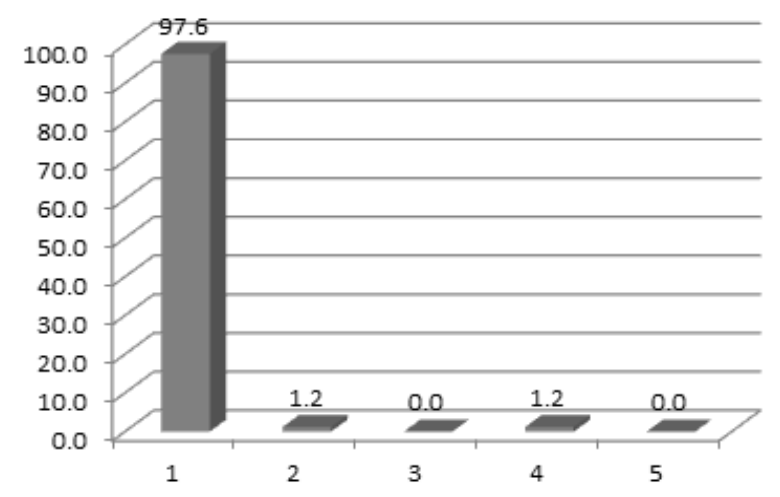

Figura 1. Red social preferida.

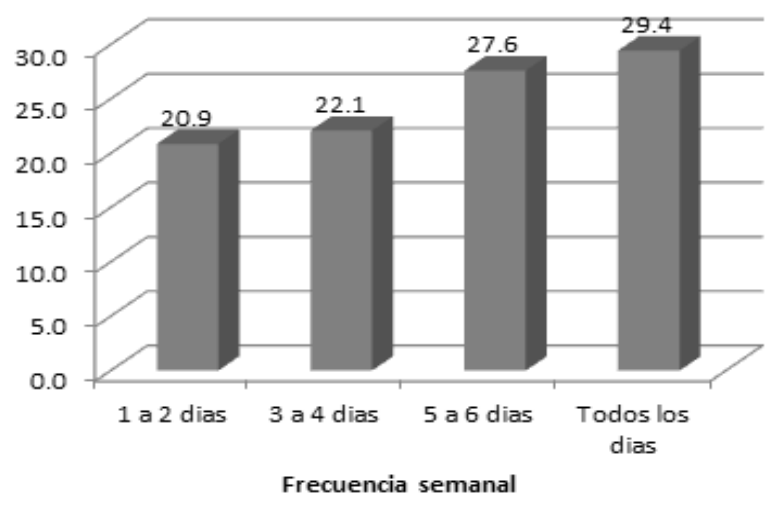

Figura 2. Frecuencia semanal de uso de redes.

Como muestra la Fig. 2, se preguntó a los estudiantes la cantidad de días a la semana que tienen acceso a las redes sociales. Cabe señalar que la mayoría revisa todos los días su red o casi todos los días, esto es revelador ya que se deduce que es una gran fuente de información y socialización para ellos; la creación de una plataforma paralela a su vida física, llena de relaciones sociales e intercambios de información con otras personas.

Sin embargo, al preguntar por la cantidad de horas dedicadas cada día, la mayoría no reconoció dedicar demasiadas horas a frecuentar de manera continua la red. (cómo se parecía en la Fig.3) Esto puede deberse a que se usa como un referente de información breve y rápida. $\mathrm{O}$ porque no perciben el paso del tiempo, lo cual es señalado por diversos investigadores, relacionando a la adicción a la dopamina generada por el recorrido y la expectativa en la red.

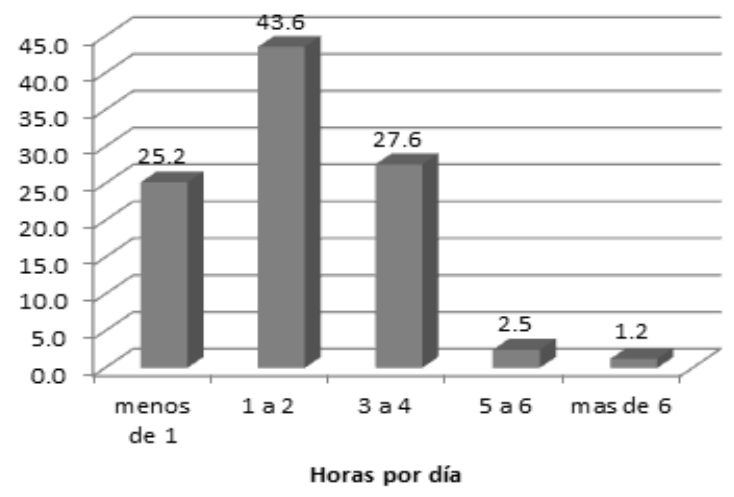

Figura 3. Horas diarias de uso de las redes sociales.

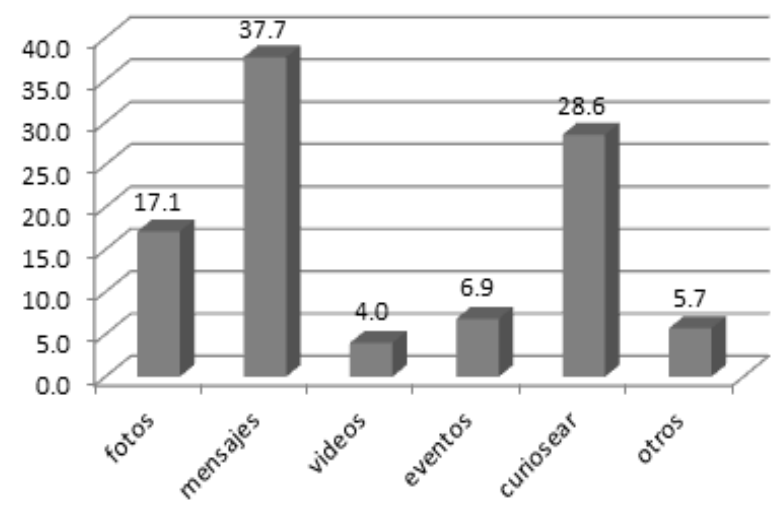

Figura 4. Aplicación favorita en las redes sociales.

Al preguntar cuál de las aplicaciones de la red usada es la de mayor frecuencia, los estudiantes reconocieron que las actividades que más realizan son intercambiar mensajes con personas conocidas y revisar los perfiles de las personas; entiéndase fotografías e información. Esto reafirma que la red social es para los jóvenes un medio de comunicación y entretenimiento. Se aprecia en la Fig.4.

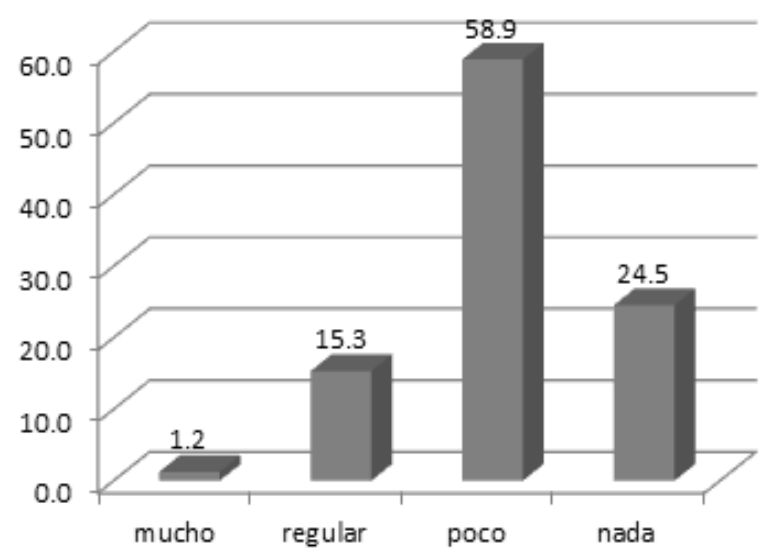

Figura 5. Influencia percibida en la forma de hablar. 
Como se ve en la Fig. 5, la gran mayoría de la muestra no considera que las redes sociales influyan demasiado en su forma de hablar. Si bien, casi un $60 \%$ considera que si influye aunque poco, esa aceptación deja entrever algo de influencia.

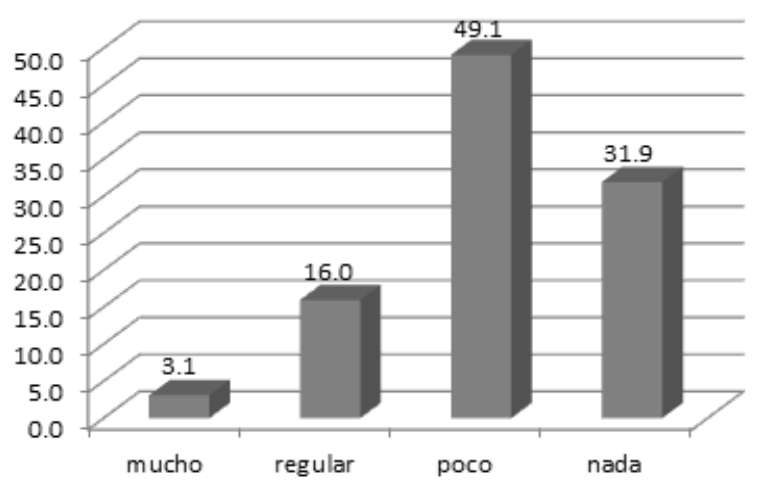

Figura 6. Porcentaje de influencia percibida en la forma de escribir.

Algo similar ocurre con la influencia percibida sobre la escritura de los estudiantes (Fig.6). Un 31.9\% considera que no influye en absoluto, sin embargo el resto de estudiantes si acepta un nivel de influencia. Es importante notar que un $3.1 \%$ sostiene que su escritura ha sido muy influenciada por el uso de las redes. Es bastante común el uso de abreviaturas y a veces vicios de economía lingüística (como obviar tildes, usar apócopes o iniciales de frases convencionales con fines comunicativos.

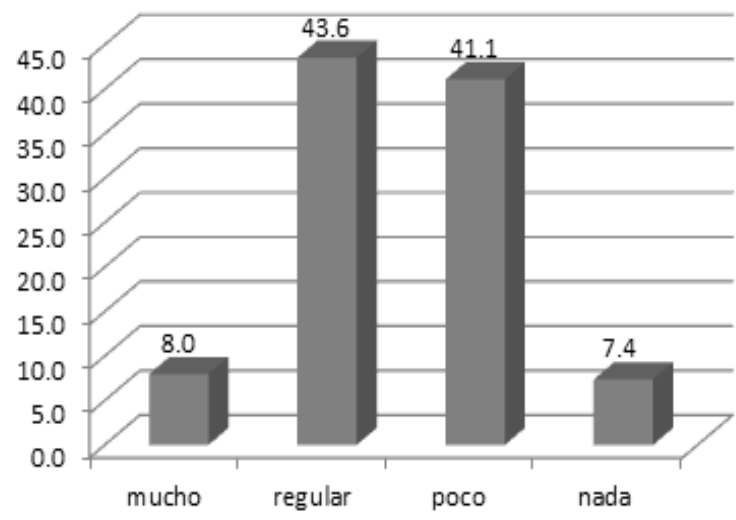

Figura 7. Nivel de información percibido.

Ocurre un fenómeno contrario cuando se pregunta por el nivel de información que reciben a través de la red, ya que sólo un $7.4 \%$ considera que no se informa a través de su red social, y más del 50\% acepta que recibe de regular a mucha información: esto reafirma las capacidades comunicacionales e informativas de las redes sociales, más allá de la influencia en los hábitos o comportamientos de los usuarios. La diferencia se aprecia en la Fig. 7.

Sorprendentemente, como puede verse en la Fig. 8, hay un $12.3 \%$ de los jóvenes encuestados que no consideran que la red influya en sus relaciones sociales. Este dato sorprende porque es contradictorio con su uso directo de las redes, que fundamentalmente son relaciones sociales. $\mathrm{O}$ no perciben el contacto vía red como relaciones sociales o les cuesta reconocer que la red influya en ellas. Sin embargo, son éstos los que menos usan la red. Igualmente hay que tener en cuenta que más del 50\% aceptan una influencia considerable (mucho o regular)

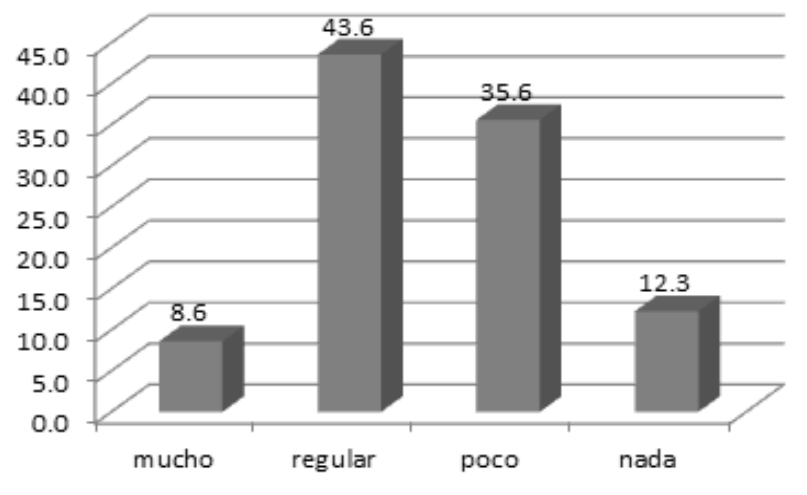

Figura 8. Influencia en las relaciones sociales.

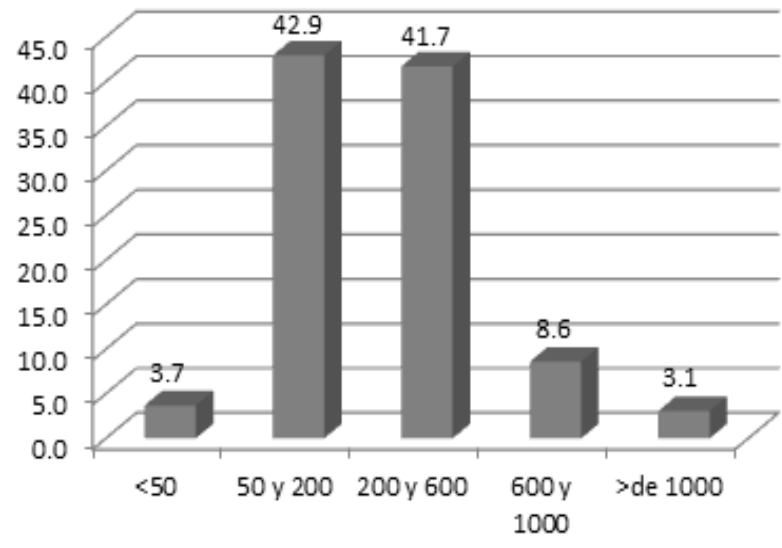

Figura 9. Cantidad de amigos o seguidores.

En la Fig. 9, se puede apreciar como la gran mayoría de usuarios tiene entre 50 y 600 personas afiliadas a su red social virtual. Esto significa que, virtualmente, nuestras redes son bastante más numerosas que las redes físicas que establecemos en la vida material. Por lo cual podemos decir que los usuarios amplían sus contactos sociales a través de las redes, pero no consideran este crecimiento de contactos sociales como un entorno real. Un caso anecdótico que confesaba uno de los jóvenes entrevistados, es que tiene como amigos en su red, mucha gente que cuando se encuentra con ella en un entorno real, no lo saluda ni intercambia ningún diálogo con él. Sin embargo es saludado en su cumpleaños por estas personas en el mundo virtual. Cabría preguntarse si estas personas forman o no parte de su entorno social, lo 
cual implicaría determinar y redefinir a lo que los jóvenes llaman entorno social y sus límites.

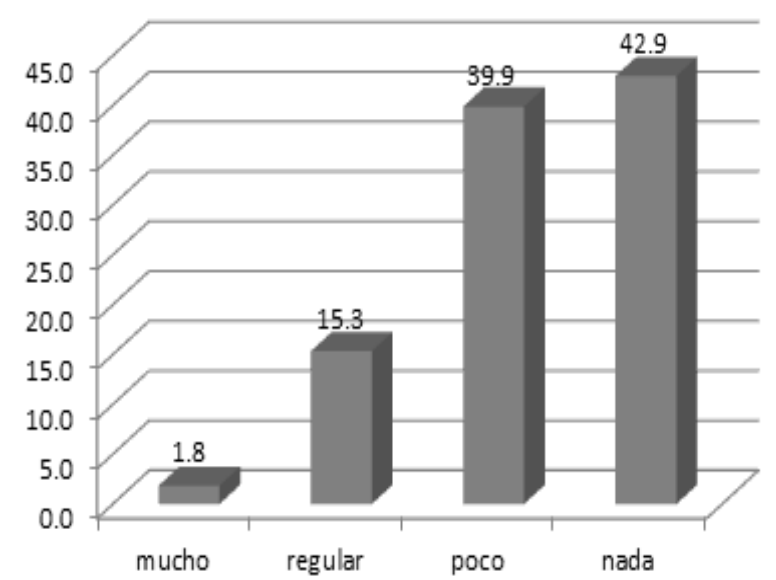

Figura 10. Influencia en los valores.

Por otro lado, como se ve en la Fig. 10, los jóvenes consideran en su mayoría que la red social que usan no influye en sus valores, esto podría traducirse también como que la red no transmite valores o como que los jóvenes no consideran valores a los transmitidos por la red que usan. Sin embargo esto pareciera contradecirse con la cantidad de frases, memes, y reflexiones de autoayuda que circulan en las redes sociales. La observación de las redes, en especial el Facebook, denota que los usuarios suelen usarla precisamente para difundir sus valores propios o aquellos que desean compartir.

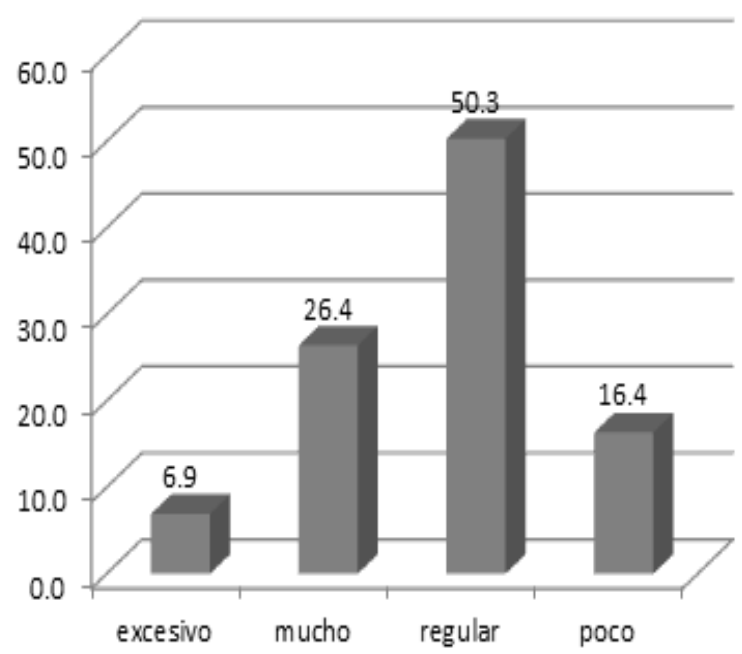

Figura 11. Consideración del tiempo que usan las redes.

Otro dato interesante -que se aprecia en la Fig.11- es que los jóvenes estudiantes, en su mayoría, no consideran que usan demasiado tiempo en las redes, digamos que su percepción al respecto se encuentra en un nivel medio. Aunque un importante $36.3 \%$ considera que invierte excesivo o mucho tiempo en éstas.

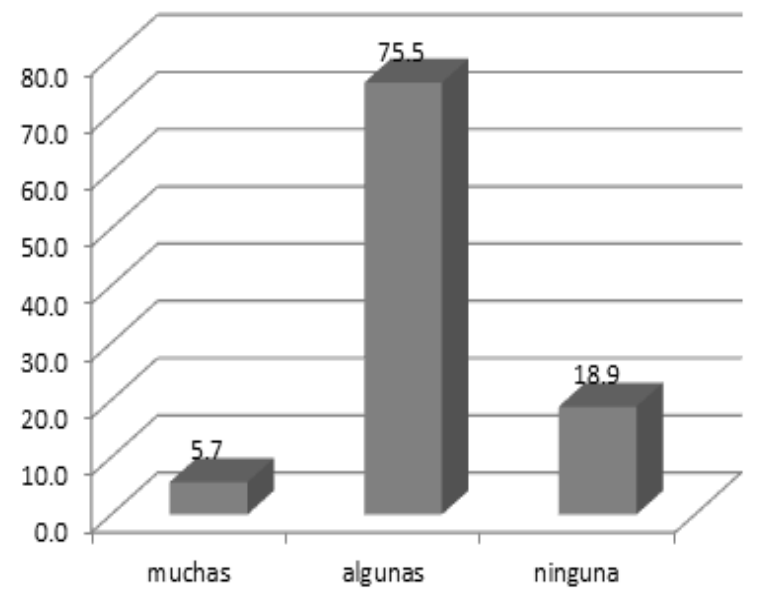

Figura 12. Aprendizaje de palabras nuevas.

Los jóvenes reconocen que han aprendido algunas palabras nuevas en la red-como se ve en la Fig. 12- que usan en su vida cotidiana, esto considera una influencia aceptada. Lo cual refuerza la idea de influencia sobre el lenguaje oral y escrito, registrado en una pregunta anterior.

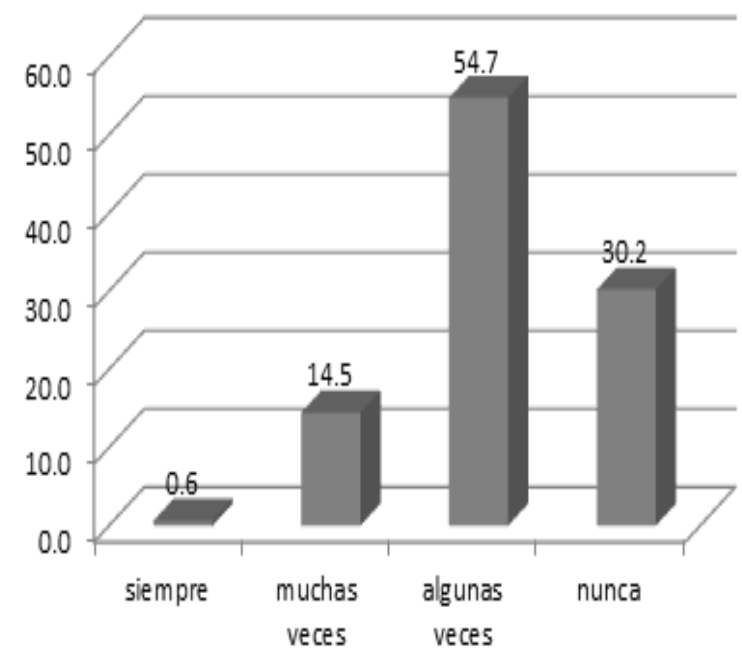

Figura 13. Uso de la escritura de las redes, fuera de las redes.

A pesar que en un ítem anterior los estudiantes no consideran que las redes hayan influido mucho en su forma de escritura, se aprecia en la Fig. 13, que la mayoría (69.8\%) consideran en esta pregunta que si usan estas redes en alguna medida. Es decir, aceptan que su forma de escribir fuera de la red, está influenciada por las formas textuales que se usan en las redes, principalmente en el Facebook. 


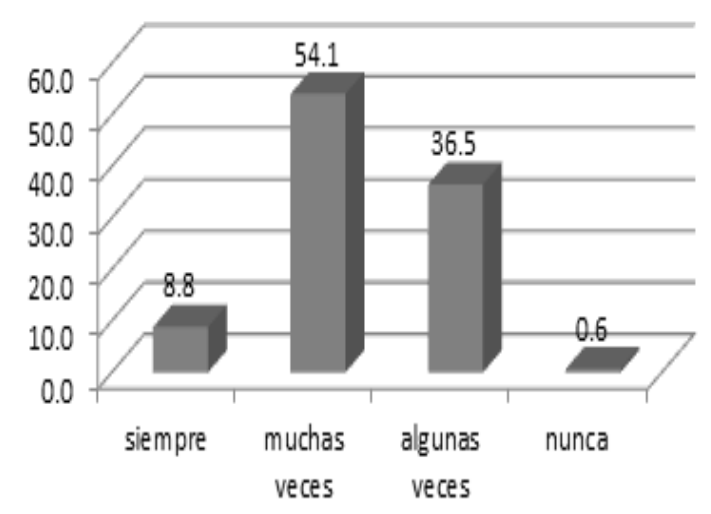

Figura 14. Novedades y datos que los estudiantes encuentran.

La Fig. 14. Reafirma un ítem anterior del mismo indicador. Los jóvenes reconocen una enorme cantidad de información que reciben a través de las redes sociales virtuales. Casi la totalidad (99.4\%) aceptan que la red les da información y datos de interés, esto se correlaciona con la frecuencia de uso y acceso.

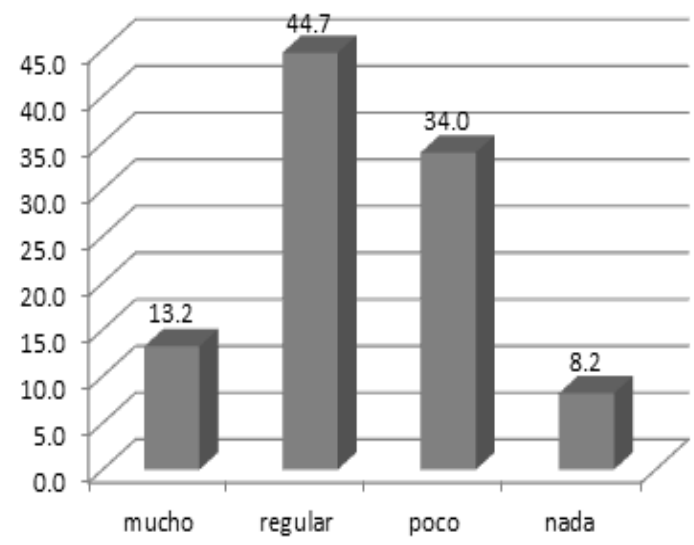

Figura 15. Mejora de sus relaciones sociales.

Casi la mitad de los encuestado en la muestra, consideran que su red social mejora sus relaciones sociales, esto va de la mano con la idea de considerar a su red social como un círculo de amigos con los cuales intercambia mensajes e información. Como se aprecia en la Fig. 15, los jóvenes reconocen la influencia que probablemente sea en realidad mayor a la reconocida.

Contrariamente a lo mencionado antes, se aprecia en la Fig. 16 que, más del $40 \%$ de los jóvenes consideran que la red social que usan les enseña valores positivos, sobre todo a través de las campañas solidarias que se difunden vía Facebook en la cual colaboran o participan de manera sencilla (dando un like) lo cual permite deducir que la red social juega un papel de catarsis frente a las presiones sociales y da a los jóvenes una percepción de que colaboran con causas sociales con un solo click

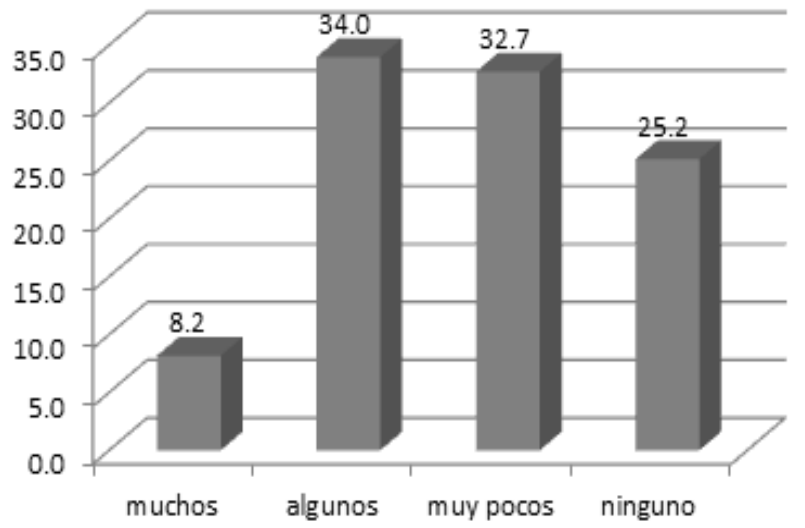

Figura 16. Aprendizaje de valores positivos.

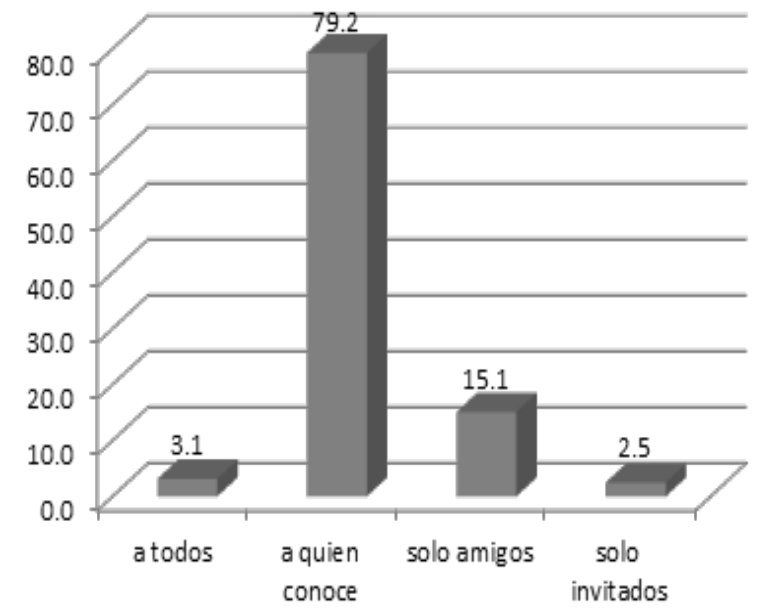

Figura 17. Aceptación en la red social.

Por último, en la Fig. 17, se aprecia que un mayoritario número de jóvenes se resiste a aceptar en su red a personas desconocidas. Sin embargo, en el otro extremo, son muy pocas los estudiantes que usan su red de manera privada, aceptando sólo a un número reducido de invitados que él mismo selecciona. Esto reafirma el uso de la red sobre los contactos sociales y el aumento de la popularidad, o la sensación sicológica de que se es más popular.

Queda demostrada la hipótesis de trabajo. Las redes sociales virtuales, influyen significativamente en los valores culturales de los jóvenes de la UNALM, considerados en el presente estudio.

\section{Conclusiones}

Las redes sociales virtuales juegan un enorme papel en la socialización de los jóvenes estudiantes, estas redes se han convertido en una influyente fuente de información y entretenimiento que usan sin darse mucha cuenta de la importancia en sus vidas reales.

A pesar que les cuesta reconocer que las redes influyen en su lenguaje escrito y oral, esta influencia existe, principalmente a través de palabras sacadas del entorno 
virtual, tanto formal como informalmente.

Los valores éticos de la sociedad se filtran a través de las redes, y éstas últimas se convierten en factores difusores de valores, relacionados principalmente a la solidaridad y a la consciencia social.

Las redes sociales son una nueva forma de comunicación que permite compartir gustos, intereses e interactuar. Se caracteriza por ser cuantitativa, de poca inversión y de alto alcance.

$\mathrm{Su}$ influencia ayuda a que superemos la individualidad, estimulemos la creatividad y la participación en grupos de interés, pero también influye negativamente aislando a la persona de la realidad.

Muchas veces la redes sociales son consideradas negativas para la formación de los jóvenes debido a la excesiva atención que se le brinda, tachándolo de adictivo; sin embargo, esta generación de jóvenes ha encontrado la manera de entrar en un equilibrio con su mundo interno es decir que a través de estos sitios web es que se puede entablar una comunicación más sincera con su interlocutor(es), más libre, sin miedo a la posible reacción que podría darse por parte de las personas con quien se está comunicando.

Las redes sociales pueden valiosamente usadas con fines académicos, empresariales y educativos, este potencial puede hacerse realidad gracias a la enorme importancia de su lenguaje para los jóvenes estudiantes.

\section{Literatura citada}

Feshbach, S. 1991. Television and Agression. Ed. UMI, 1991.

Gomez, C. 1998. Comunicación y educación en la era digital. Ed. Diana (1998)

Hernandez, S. R. 2006. Metodología de la Investigación. Ed. McGraw- Hill Interamericana. México, 2006.

Manrique, N. 1996. Los apocalípticos de Internet. El Peruano, Miércoles 21 de agosto de 1996.

Oliva M. C. 2014. El periodismo digital y sus retos en la sociedad global y del conocimiento. Aposta, revista de ciencias sociales. Madrid, junio 2014

Small, G. 2009. El Cerebro Digital. Ed. Urano, España, 2009.

Unger, T. 2006. La revolución del acceso a medios. El Comercio, martes 1 de agosto del 2006.

Zamora, M. 2006. Redes Sociales en internet (2006). Maestros del Web. http://www.maestrosdelweb.com/ redessociales/ 